\title{
On Dual Configurational Forces
}

\author{
SHAOFAN LI ${ }^{\star}$ and ANURAG GUPTA \\ Department of Civil and Environmental Engineering, University of California, Berkeley, \\ CA 94720, USA.E-mail: li@ce.berkeley.edu
}

Received 6 January 2005; in revised form 9 December 2005

\begin{abstract}
The dual conservation laws of elasticity are systematically re-examined by using both Noether's variational approach and Coleman-Noll-Gurtin's thermodynamics approach. These dual conservation laws can be interpreted as the dual configurational force, and therefore they provide the dual energy-momentum tensor. Some previously unknown and yet interesting results in elasticity theory have been discovered. As an example, we note the following duality condition between the configuration force (energy-momentum tensor) $\mathcal{P}$ and the dual configuration force (dual energy-momentum tensor) $\mathcal{L}$,
\end{abstract}

$$
\mathcal{P}-\mathcal{L}=(\mathbf{P}: \mathbf{F}) \mathbf{1}-\nabla(\mathbf{P} \cdot \mathbf{x}) .
$$

This and other results derived in this paper may lead to a better understanding of configurational mechanics and therefore of mechanics of defects.

Mathematics Subject Classification (2000): 35L65, 37K05, 49S05, 58E30

Key words: complementary variational principles, configuration force, conservation laws, energymomentum tensor.

\section{Introduction}

Since Eshelby's pioneer work [3, 4] on the energy-momentum tensor, the subject of configurational force has been at the center of attention of continuum mechanics and material science. The configurational forces are associated with the relative change in the energy with respect to various configurational rearrangements. Such rearrangements can occur within a lattice as a motion of a dislocation or due to propagation of a crack. For an overview of the various applications of configurational forces, the reader is referred to the texts by Maugin [18], Gurtin [10], and Kienzler and Herrmann [11] and a review paper by Maugin [19]. There has been a considerable amount of research regarding the presence and applicability of Eshelby's energy-momentum tensor, but there is a scarcity of results regarding its thermodynamical dual. This work is aimed at a systematic study of obtaining dual configurational forces.

Eshelby [4] had already anticipated the existence of a complementary energymomentum tensor (referred to as the dual energy-momentum tensor in this

\footnotetext{
$\star$ Corresponding author.
} 
paper), but it was Bui [1], who first derived a complementary path-independent integral, and provided some physical interpretations. Bui's I-integral has been interpreted as the dual of the Rice's J-integral [29]. Sun [30] and Li [16] used Noether's theorem [22] to obtain different sets of dual conservation laws in linear elastostatics. Soon afterwards, Moran and Shih [20,21] studied these dual pathindependent integrals under a thermodynamical setting. In finite deformation, a counterpart of Bui's path-independent integral was derived by Trimarco and Maugin [31]. More recently, Li [14] derived another set of dual conservation laws of planar elasticity via stress function formalism. A class of conservation laws expressed purely in terms of the stress tensor for the case of 3D linear and isotropic elasticity has been obtained by Li et al. [15].

Overall in the past 50 years, the research on dual path-independent integrals is scarce. One reason for this may be due to a general perception that the dual pathindependent integrals do not provide any new physical content. As Moran and Shih [21] commented in their 1987 paper,

“.... Bui has shown that the energy and complementary energy release rates are equal. We establish in a direct manner that Bui's observation is valid for quite general material response and for all dual crack tip integrals under discussion. The implication is that no new information can be extracted from complementary integrals which is not already provided by their counterparts. However, in certain circumstances, the pair of integrals, referred to as dual integrals, may be employed to obtained a bound for the crack tip parameter. ...."

Despite the validity of this statement, ambiguity remains in the subject. We show that under the infinitesimal deformation, Bui's I-integral does not belong to the variational symmetry of the pure complementary strain energy principle, but rather is an outcome of the translational invariance of a mixed variational principle - the Hellinger-Reissner principle. Moreover, in finite deformation, the existence of purely stress based complementary variational principles has been a well-known debate existing for over several decades (see $[5,6,23]$ ). There are several complementary variational principles in finite elasticity, but their variational symmetries have never been carefully studied.

In this paper, a systematic effort is made to re-examine the dual invariant integrals in elasticity. We apply Noether's theorem [22] to examine the invariant conditions for various complementary energy functionals in elasticity. We first examine the invariant integrals resulting from the Hellinger-Reissner principle in linear elasticity, and we then study the translational invariance of complementary energy principles in finite elasticity, which include the Hellinger-Reissner principle, the Levinson principle, the Fraeijs de Veubeke principle, and the most recent principle introduced by Gao $[6,7]$.

In the end, as an alternative approach, we follow the procedure initiated by Gurtin [9], but use the Gibbs free energy instead of the Helmhotz free energy, 
and as a result we obtain the expression for the dual configurational force without using any variational principle.

\section{Linear Elastostatics}

We start with the variational symmetry approach, which can be summarized by stating an appropriate version of the Noether's first theorem without proof (see [25-27]) below,

THEOREM 2.1. If the fundamental integral, $\Pi\left(u_{i}, \sigma_{i j}, \sigma_{i j, k}\right)$, involving the Lagrangian $L_{\Omega}$ is invariant under the r-parameter transformations, then the following conservation laws hold

$$
\frac{d}{d x_{k}}\left[\left(L_{\Omega} \delta_{k \ell}-\sigma_{i j, \ell} \frac{\partial L_{\Omega}}{\partial \sigma_{i j, k}}\right) \varphi_{\ell \alpha}+\frac{\partial L_{\Omega}}{\partial \sigma_{i j, k}} \xi_{i j \alpha}\right]=0, \quad \alpha=1,2, \cdots, r .
$$

where, $x_{i}$ denotes the coordinate variable, $u_{i}$ represents the displacement field and $\sigma_{i j}$ denotes the symmetric Cauchy stress tensor. $\delta_{k l}$ represents the usual Kronecker delta operator. The Einstein summation rule for repeated indices is used. We denote $\varphi_{i \alpha}, \xi_{i j \alpha}$ and $\eta_{i \alpha}$ as the infinitesimal generators (see below for their definition) corresponding to the coordinate variable, the stress tensor and the displacement vector, respectively.

We now apply the above theorem to the following two field HellingerReissner functional [28, 33],

$$
\begin{aligned}
\Pi\left(u_{i}, \sigma_{i j}, \sigma_{i j, k}\right)= & -\int_{\Omega}\left\{\frac{1}{2} \sigma_{i j} D_{i j k \ell} \sigma_{k \ell}+\sigma_{j i . j} u_{i}\right\} d V \\
& +\int_{\Gamma_{u}} t_{i} \tilde{u}_{i} d S+\int_{\Gamma_{t}}\left(t_{i}-\tilde{t}_{i}\right) u_{i} d S .
\end{aligned}
$$

Where the fourth order tensor $D_{i j k l}$ is the elastic compliance tensor, such that $\varepsilon_{i j}=D_{i j k l} \sigma_{k l}$, where $\varepsilon_{k l}$ is the symmetrical strain tensor. The vector $t_{i}$ denotes the traction field, and the vectors $\tilde{u}_{i}$ and $\tilde{t}_{i}$ are the prescribed displacement and traction. For the purpose of our discussion, we only consider two symmetries corresponding to the rigid body translation and rotation:

\subsection{COORDINATE TRANSLATION}

Let

$$
\bar{x}_{i}=x_{i}+\epsilon_{i},
$$




$$
\begin{gathered}
\bar{\sigma}_{i j}=\sigma_{i j}, \\
\bar{u}_{i}=u_{i},
\end{gathered}
$$

where $\epsilon$ is a small parameter. The corresponding generators of infinitesimal transformations are

$$
\begin{aligned}
& \varphi_{i \alpha}=\left.\frac{\partial \bar{x}_{i}}{\partial \epsilon_{\alpha}}\right|_{\boldsymbol{\epsilon}=0}=\delta_{i \alpha}, \quad i, \alpha=1,2,3, \\
& \xi_{i j \alpha}=\left.\frac{\partial \bar{\sigma}_{i j}}{\partial \epsilon_{\alpha}}\right|_{\boldsymbol{\epsilon}=0}=0, \\
& \eta_{i \alpha}=\left.\frac{\partial \bar{u}_{i}}{\partial \epsilon_{\alpha}}\right|_{\boldsymbol{\epsilon}=0}=0 .
\end{aligned}
$$

Therefore we have the following conservation law,

$$
\frac{d E_{k \alpha}^{c}}{d x_{k}}=0, \quad \alpha=1,2,3,
$$

where

$$
E_{k \alpha}^{c}=-\left(\frac{1}{2} \sigma_{i j} D_{i j m n} \sigma_{m n}+\sigma_{j i, j} u_{i}\right) \delta_{k \alpha}+\sigma_{i k, \alpha} u_{i}
$$

REMARK 2.1. Suppose $\sigma_{i j}$ is statically admissible, i.e., $\sigma_{j i . j}=0$. Choose $\alpha=1$. One can then obtain the following invariant integral:

$$
I=\oint_{\Gamma}\left(-W^{c} n_{1}+u_{i} \sigma_{i k, 1} n_{k}\right) d S,
$$

which is the Bui's I-integral. $W^{c}=\frac{1}{2} \sigma_{i j} D_{i j m n} \sigma_{m n}$ is the complementary energy density for a linear elastic material.

REMARK 2.2 The fundamental integrals given by Sun [30] or by Li [16] have no clear physical meanings. An obvious motive in construction of those integrals is to match Bui's I integral. As shown above, the correct way to derive Bui's Iintegral and the dual Eshelby energy-momentum tensor should be based on the Hellinger-Reissner two field principle.

REMARK 2.3. Define $E_{k \alpha}^{c}$ as the dual Eshelby energy-momentum tensor. Combining $E_{k \alpha}^{c}$ with the Eshelby's energy-momentum tensor (e.g., Knowles and Sternberg [12]),

$$
E_{k \alpha}=W \delta_{k \alpha}-\sigma_{i k} u_{i, \alpha},
$$






where $W$ is the energy density for a linear elastic material, we have

$$
\begin{aligned}
E_{k \alpha}-E_{k \alpha}^{c} & =\left(W+W^{c}\right) \delta_{k \alpha}-\frac{d}{d x_{\alpha}}\left(\sigma_{i k} u_{i}\right) \\
& =\sigma_{i j} \epsilon_{i j} \delta_{k \alpha}-\frac{d}{d x_{\alpha}}\left(\sigma_{i k} u_{i}\right) .
\end{aligned}
$$

This suggests that the tensor $E_{k \alpha}$ and tensor $E_{k \alpha}^{c}$ form a conjugate pair of the above differential transformation, which is different from the scalar form of the Legendre transformation.

\subsection{COORDINATE ROTATION}

Let

$$
\begin{aligned}
\bar{x}_{i} & =Q_{j i}(\boldsymbol{\epsilon}) x_{j}, \\
\bar{\sigma}_{i j} & =Q_{k i}(\boldsymbol{\epsilon}) \sigma_{k \ell} Q_{\ell j}(\boldsymbol{\epsilon}), \\
\bar{u}_{i} & =Q_{j i}(\boldsymbol{\epsilon}) u_{j},
\end{aligned}
$$

where the rotation matrix may be written as $Q_{i j}(\boldsymbol{\epsilon})=\delta_{i j}+\varepsilon_{i j k} \epsilon_{k}+o(\boldsymbol{\epsilon})$, and $\varepsilon_{i j k}$ is the permutation symbol.

The corresponding infinitesimal generators are then given as following:

$$
\begin{aligned}
& \varphi_{i \alpha}=\left.\frac{\partial \bar{x}_{i}}{\partial \epsilon_{\alpha}}\right|_{\boldsymbol{\epsilon}=0}=\varepsilon_{j i \alpha} x_{j}, \quad \alpha=1,2,3, \\
& \xi_{i j \alpha}=\left.\frac{\partial \bar{\sigma}_{i j}}{\partial \epsilon_{\alpha}}\right|_{\boldsymbol{\epsilon}=0}=\varepsilon_{l i \alpha} \sigma_{l j}+\varepsilon_{l j \alpha} \sigma_{l i}, \\
& \eta_{i \alpha}=\left.\frac{\partial \bar{u}_{i}}{\partial \epsilon_{\alpha}}\right|_{\boldsymbol{\epsilon}=0}=\varepsilon_{j i \alpha} u_{j} .
\end{aligned}
$$

The corresponding conserved quantities are then given as:

$$
R_{k \alpha}^{c}=-W^{c} \varepsilon_{j k \alpha} x_{j}+\sigma_{k j, l} u_{j} \varepsilon_{m l \alpha} x_{m}-u_{j}\left(\varepsilon_{l k \alpha} \sigma_{l j}+\varepsilon_{l j \alpha} \sigma_{l k}\right) .
$$

It can be verified (by taking a divergence) that the above quantity is conserved only in the case of isotropy, i.e., when stress and strain tensors are coaxial. We also recall here the corresponding conservation law obtained via the usual variational principle [12],

$$
R_{k \alpha}=W \varepsilon_{j k \alpha} x_{j}-\sigma_{k j} u_{j, l} \varepsilon_{m l \alpha} x_{m}+u_{j} \varepsilon_{j i \alpha} \sigma_{i k} .
$$


We finally note that the following relation holds between these two quantities:

$$
R_{k \alpha}-R_{k \alpha}^{c}=\left(W^{c}+W\right) \varepsilon_{j k \alpha} x_{j}-\left(\sigma_{k j} u_{j} \varepsilon_{m \ell \alpha} x_{m}\right)_{, \ell}+u_{j} \sigma_{\ell j}\left(\varepsilon_{m k \alpha} x_{m}\right)_{, \ell} .
$$

\section{Finite Elasticity}

Based on mathematical principles, the dual configuration force should be related to the translational invariance of a complementary energy variational principle. As mentioned above, the complementary strain energy principle in finite elasticity has been a controversial topic for many years (see $[5,8]$ for historical notes). Although there were quite a few complementary energy principles in finite elasticity, until recent contribution by Gao [6], none of these complementary principles was regarded as a pure complementary variational principle (for the reasons that will be explained later in the paper). This may have had a definite setback on the study of dual conservation laws in finite elasticity as well. The only documented literature that we were able to find is by Trimarco and Maugin [31]. In this section, we derive the dual configuration force from several complementary variational principles in finite elasticity.

Consider a typical particle point with position $\mathbf{X}$ in a three dimensional reference configuration $\Omega$, which under deformation $\chi$ is transformed to a position $\mathbf{x}$ in the current configuration,

$$
\mathbf{x}=\chi(\mathbf{X})=\mathbf{X}+\mathbf{u}(\mathbf{X}), \quad \mathbf{X} \in \Omega
$$

where $\mathbf{u}$ is the displacement vector. The gradient of the deformation $\mathbf{F}(\mathbf{X})$ is defined by $d \chi=\mathbf{F} d \mathbf{X}$. Thus,

$$
\mathbf{F}(\mathbf{X})=\operatorname{grad} \chi(\mathbf{X})=F_{i a} \mathbf{e}_{i} \otimes \mathbf{e}_{a}, \quad F_{i a}=\frac{\partial \chi_{i}}{\partial X_{a}}, \quad i, a \in\{1,2,3\} .
$$

The right and left Cauchy-Green tensors are defined as $\mathbf{C}=\mathbf{F}^{T} \mathbf{F}$ and $\mathbf{b}=\mathbf{F F}^{T}$, respectively, where the superscript $T$ represents the transpose. The inverse of the right Cauchy-Green tensor, referred as the Piola deformation tensor, is denoted by $\mathbf{B}=\mathbf{C}^{-1}=\mathbf{F}^{-1} \mathbf{F}^{-T}$. We define the Green-Lagrange strain tensor as

$$
\mathbf{E}=\frac{1}{2}(\mathbf{C}-\mathbf{1})=\frac{1}{2}\left[\nabla \mathbf{u}+\nabla \mathbf{u}^{T}+\nabla \mathbf{u} \nabla \mathbf{u}^{T}\right] .
$$

The deformation gradient admits a unique polar decomposition: $\mathbf{F}=\mathbf{R} \mathbf{U}$, where $\mathbf{R} \in S O(3)$ and $\mathbf{U}$ is the (right) stretch tensor. Hence, $\mathbf{C}=\mathbf{F}^{T} \mathbf{F}=\mathbf{U}^{2}$. Next we introduce various stress measures. We denote $\mathbf{P}$ as the first Piola-Kirchhoff stress tensor, $\mathbf{S}$ as the second Piola-Kirchhoff stress tensor, $\sigma$ as the Cauchy stress tensor, $\boldsymbol{\tau}$ as the Kirchhoff tensor, and $\mathbf{T}$ as the Biot stress tensor (also known as Jaumann stress tensor). The Cauchy stress can be physically interpreted as well 
as derived from the traction force $\mathbf{t}$ on the deformed configuration with the normal $\mathbf{n}$, i.e., $\sigma \mathbf{n}=\mathbf{t}$. These stress tensors are related to each other as,

$$
\begin{aligned}
\mathbf{P} & =\mathbf{F S}, \\
\sigma & =J^{-1} \mathbf{F S F}^{T}, \\
\boldsymbol{\tau} & =\mathbf{F} \mathbf{S F}^{T}, \\
\mathbf{T} & =\frac{1}{2}(\mathbf{S U}+\mathbf{U S})=\frac{1}{2}\left(\mathbf{P}^{T} \mathbf{R}+\mathbf{R}^{T} \mathbf{P}\right),
\end{aligned}
$$

where $J=\operatorname{det} \mathbf{F}$.

We assume the material under consideration to be hyperelastic. Therefore the material possesses a stored-energy function $\Sigma$ (per unit volume in the reference configuration), which may be regarded as a function of any strain measure, for example $\mathbf{E}, \mathbf{U}$, etc. Stress measures are taken as conjugates to various strain measures. We then have

$$
\begin{aligned}
& \mathbf{S}(\mathbf{E})=\frac{\partial \Sigma}{\partial \mathbf{E}}, \\
& \mathbf{P}(\mathbf{F})=\frac{\partial \Sigma}{\partial \mathbf{F}}, \\
& \mathbf{T}(\mathbf{U})=\frac{\partial \Sigma}{\partial \mathbf{U}} .
\end{aligned}
$$

Thus, various complementary energy density functions can be defined [8]:

$$
\begin{aligned}
& \Sigma^{c}(\mathbf{S})=\mathbf{S}: \mathbf{E}(\mathbf{S})-\Sigma(\mathbf{E}(\mathbf{S})), \\
& \tilde{\Sigma}^{c}(\mathbf{P})=\mathbf{P}: \mathbf{F}(\mathbf{P})-\Sigma(\mathbf{F}(\mathbf{P})), \\
& \tilde{\Sigma}^{c}(\mathbf{T})=\mathbf{T}: \mathbf{U}(\mathbf{T})-\Sigma(\mathbf{U}(\mathbf{T})),
\end{aligned}
$$

where an appropriate invertibility is needed in all the relations (see [24] for details regarding invertibility of various conjugate pairs). We would like to elaborate on (34). Under an unique decomposition, $\mathbf{F}$ determines strain (and therefore stress) uniquely. But a stress state may correspond to the same strain state under different rotations and therefore invertibility of $\mathbf{P}(\mathbf{F})$ is not always 
guaranteed. Equation (34) is valid under the assumption that $\mathbf{P}(\mathbf{F})$ is invertible, which implies that

$$
\mathbf{F}(\mathbf{P})=\frac{\partial \Sigma^{c}}{\partial \mathbf{P}}
$$

If $\mathbf{P}(\mathbf{F})$ is not invertible we can rewrite (35) using (29) as

$$
\tilde{\Sigma}^{c}(\mathbf{T}(\mathbf{P}, \mathbf{R}))=\mathbf{P}:[\mathbf{R} \cdot \mathbf{U}(\mathbf{T})]-\Sigma(\mathbf{U}(\mathbf{T})) .
$$

Note that both $\mathbf{P}$ and $\mathbf{R}$ are simultaneously regarded as independent variables. The invertibility of the relation (32) can be argued in a much broader space than that of the relation (31) (See Chapter 6 of [24] for details).

We now examine the translational symmetry of some main complementary variational principles of finite elasticity.

\subsection{THE HELLINGER-REISSNER PRINCIPLE}

The Hellinger-Reissner principle involves the following functional [28]:

$$
\begin{aligned}
\breve{\Pi}_{H R}^{c}(\mathbf{u}, \mathbf{S})= & -\int_{\Omega}\left\{\Sigma^{c}(\mathbf{S})+\frac{1}{2}\left[(\nabla \mathbf{u})^{T}(\nabla \mathbf{u})\right]: \mathbf{S}+\mathbf{u} \cdot[\nabla \cdot(\mathbf{F S})]\right\} d V \\
& +\int_{\Gamma_{u}} \hat{\mathbf{u}} \cdot(\mathbf{F S} \cdot \mathbf{N}) d A+\int_{\Gamma_{t}} \mathbf{u} \cdot\left(\mathbf{F S} \cdot \mathbf{N}-\hat{\mathbf{t}}_{N}\right) d A,
\end{aligned}
$$

where the vectors with $\mathrm{a}^{\wedge}$ are imposed quantities. Here the Lagrangian is given by

$$
L(\mathbf{u}, \mathbf{S}, \nabla \mathbf{u}, \nabla \mathbf{S}, \nabla \nabla \mathbf{u})=-\Sigma^{c}(\mathbf{S})-\frac{1}{2}\left[(\nabla \mathbf{u})^{T}(\nabla \mathbf{u})\right]: \mathbf{S}-\mathbf{u} \cdot[\nabla \cdot(\mathbf{F S})],
$$

where $\mathbf{F}=\mathbf{1}+\nabla \mathbf{u}$. We now consider the following r-parameter family of invertible transformations:

$$
\begin{gathered}
\bar{X}_{a}=\bar{X}_{a}(\mathbf{X}, \mathbf{u}, \mathbf{S}, \boldsymbol{\epsilon}), \\
\bar{u}_{i}=\bar{u}_{i}(\mathbf{X}, \mathbf{u}, \mathbf{S}, \boldsymbol{\epsilon}), \\
\bar{S}_{a b}=\bar{S}_{a b}(\mathbf{X}, \mathbf{u}, \mathbf{S}, \boldsymbol{\epsilon}),
\end{gathered}
$$

where $\boldsymbol{\epsilon}=\left(\epsilon_{1}, \epsilon_{2}, \ldots, \epsilon_{r}\right)$, such that $\left.\overline{\mathbf{X}}\right|_{\boldsymbol{\epsilon}=0}=\mathbf{X},\left.\overline{\mathbf{u}}\right|_{\boldsymbol{\epsilon}=0}=\mathbf{u}$ and $\left.\overline{\mathbf{S}}\right|_{\boldsymbol{\epsilon}=0}=\mathbf{S}$. The boundary terms are assumed to remain invariant under these transformations. We can expand the transformed coordinates (40)-(42) using Taylor's theorem,

$$
\bar{X}_{a}=X_{a}+\varphi_{a p}(\mathbf{X}, \mathbf{u}, \mathbf{S}) \epsilon_{p}+o(\boldsymbol{\epsilon})
$$


ON DUAL CONFIGURATIONAL FORCES

$$
\begin{gathered}
\bar{u}_{i}=u_{i}+\lambda_{i p}(\mathbf{X}, \mathbf{u}, \mathbf{S}) \epsilon_{p}+o(\boldsymbol{\epsilon}), \\
\bar{S}_{a b}=S_{a b}+\xi_{a b p}(\mathbf{X}, \mathbf{u}, \mathbf{S}) \epsilon_{p}+o(\boldsymbol{\epsilon}),
\end{gathered}
$$

where

$$
\begin{gathered}
\varphi_{a p}(\mathbf{X}, \mathbf{u}, \mathbf{S})=\left.\frac{\partial \bar{X}_{a}}{\partial \epsilon_{p}}\right|_{\boldsymbol{\epsilon}=0}, \\
\lambda_{i p}(\mathbf{X}, \mathbf{u}, \mathbf{S})=\left.\frac{\partial \bar{u}_{i}}{\partial \epsilon_{p}}\right|_{\boldsymbol{\epsilon}=0}, \\
\xi_{a b p}(\mathbf{X}, \mathbf{u}, \mathbf{S})=\left.\frac{\partial \bar{S}_{a b}}{\partial \epsilon_{p}}\right|_{\boldsymbol{\epsilon}=0} .
\end{gathered}
$$

We can obtain the following relation, which should be satisfied by the infinitesimal generators for the functional to remain infinitesimally invariant (See [17] for details).

$$
\begin{aligned}
& \frac{\partial L}{\partial X_{i}} \varphi_{i p}+\frac{\partial L}{\partial u_{i}} \lambda_{i p}+\frac{\partial L}{\partial S_{a b}} \xi_{a b p}+\frac{\partial L}{\partial u_{i, a}}\left(\frac{d \lambda_{i p}}{d X_{a}}-u_{i, b} \frac{d \varphi_{b p}}{d X_{a}}\right) \\
& +\frac{\partial L}{\partial S_{a b, d}}\left(\frac{d \xi_{a b p}}{d X_{d}}-S_{a b, c} \frac{d \varphi_{c p}}{d X_{d}}\right)+\frac{\partial L}{\partial u_{i, a b}}\left(\frac{d}{d X_{b}} \frac{d \lambda_{i p}}{d X_{a}}-u_{i, c} \frac{d}{d X_{b}} \frac{d \varphi_{c p}}{d X_{a}}\right. \\
& \left.-\left\{u_{i, c b} \frac{d \varphi_{c p}}{d X_{a}}+u_{i, c a} \frac{d \varphi_{c p}}{d X_{b}}\right\}\right)+L \frac{d \varphi_{a p}}{d X_{a}}=0,
\end{aligned}
$$

where $i, a, b, c, d \in\{1,2,3\}$ and $p \in\{1,2, \ldots, r\}$. Furthermore we can obtain various conservation laws associated with corresponding invariant transformations as,

$$
\begin{aligned}
& \frac{d}{d X_{a}}\left\{\left[L \delta_{a b}-u_{i, b} \frac{\partial L}{\partial u_{i, a}}-S_{c d, b} \frac{\partial L}{\partial S_{c d, a}}-u_{i, b c} \frac{\partial L}{\partial u_{i, a c}}\right.\right. \\
& \left.+u_{i, b} \frac{d}{d X_{c}}\left(\frac{\partial L}{\partial u_{i, a c}}\right)\right] \varphi_{b p}+\left[\frac{\partial L}{\partial u_{i, a}}-\frac{d}{d X_{b}}\left(\frac{\partial L}{\partial u_{i, a b}}\right)\right] \lambda_{i p}+\frac{\partial L}{\partial S_{c d, a}} \xi_{c d p} \\
& \left.+\frac{\partial L}{\partial u_{i, a b}} \frac{d \lambda_{i p}}{d X_{b}}-u_{i, c} \frac{\partial L}{\partial u_{i, a b}} \frac{d \varphi_{c p}}{d X_{b}}\right\}=0 .
\end{aligned}
$$

We now consider a rigid body translation (with respect to the reference coordinate): $\bar{X}_{a}=X_{a}+\epsilon_{a}, \bar{u}_{i}=u_{i}$ and $\bar{S}_{a b}=S_{a b}$. The only non-trivial infinitesimal generator is $\varphi_{a b}=\delta_{a b}$. The condition (49) is satisfied trivially for such a transformation. Therefore we can use (50) to obtain an energy momentum tensor from Hellinger-Reissner's variational principle,

$$
\mathcal{L}_{H R}=-\tilde{\Sigma}^{c}(\mathbf{S}) \mathbf{1}-\left\{\frac{1}{2}\left[(\nabla \mathbf{u})^{T}(\nabla \mathbf{u})\right]: \mathbf{S}\right\} \mathbf{1}+\mathbf{u} \cdot[\nabla(\mathbf{F S})-(\nabla \mathbf{u})(\nabla \mathbf{S})],
$$


where we have made use of the equilibrium condition $\nabla \cdot \mathbf{F S}=0$, which arises as a one of the Euler equations from Hellinger-Reissner's variational principle.

REMARK 3.1. The above conservation law reduces to that of Bui's dual conservation law for linear elasticity. This can be seen by retaining only leading order terms from (51) and by replacing $\mathbf{S}$ by the Cauchy stress $\sigma$.

\subsection{THE LEVINSON PRINCIPLE}

Assuming that $\mathbf{P}(\mathbf{F})$ is invertible, consider the following functional [13]:

$$
\Pi_{L}^{c}(\mathbf{P}, \mathbf{x})=-\int_{\Omega}\left[\tilde{\Sigma}^{c}(\mathbf{P})+\mathbf{x} \cdot(\nabla \cdot \mathbf{P})\right] d V+\int_{\Gamma_{u}} \mathbf{P N} \cdot \hat{\mathbf{x}} d A+\int_{\Gamma_{t}}\left(\mathbf{P N}-\hat{\mathbf{t}}_{N}\right) \cdot \mathbf{x} d A
$$

and the following r-parameter family of invertible transformations,

$$
\begin{gathered}
\bar{X}_{a}=\bar{X}_{a}(\mathbf{X}, \mathbf{x}, \mathbf{P}, \boldsymbol{\epsilon}), \\
\bar{x}_{i}=\bar{x}_{i}(\mathbf{X}, \mathbf{x}, \mathbf{P}, \boldsymbol{\epsilon}), \\
\bar{P}_{i a}=\bar{P}_{i a}(\mathbf{X}, \mathbf{x}, \mathbf{P}, \boldsymbol{\epsilon}),
\end{gathered}
$$

where $\boldsymbol{\epsilon}=\left(\epsilon_{1}, \epsilon_{2}, \ldots, \epsilon_{r}\right)$, such that $\left.\overline{\mathbf{X}}\right|_{\boldsymbol{\epsilon}=0}=\mathbf{X},\left.\overline{\mathbf{x}}\right|_{\boldsymbol{\epsilon}=0}=\mathbf{x}$ and $\left.\overline{\mathbf{P}}\right|_{\boldsymbol{\epsilon}=0}=\mathbf{P}$. The boundary terms are assumed to remain invariant under these transformations. We can expand the transformed coordinates (53)-(55) using Taylor's theorem,

$$
\begin{gathered}
\bar{X}_{a}=X_{a}+\varphi_{a p}(\mathbf{X}, \mathbf{x}, \mathbf{P}) \epsilon_{p}+o(\boldsymbol{\epsilon}), \\
\bar{x}_{i}=x_{i}+\lambda_{i p}(\mathbf{X}, \mathbf{x}, \mathbf{P}) \epsilon_{p}+o(\boldsymbol{\epsilon}), \\
\bar{P}_{i a}=P_{i a}+\xi_{i a p}(\mathbf{X}, \mathbf{x}, \mathbf{P}) \epsilon_{p}+o(\boldsymbol{\epsilon}),
\end{gathered}
$$

where

$$
\begin{gathered}
\varphi_{a p}(\mathbf{X}, \mathbf{x}, \mathbf{P})=\left.\frac{\partial \bar{X}_{a}}{\partial \epsilon_{p}}\right|_{\boldsymbol{\epsilon}=0}, \\
\lambda_{i p}(\mathbf{X}, \mathbf{x}, \mathbf{P})=\left.\frac{\partial \bar{x}_{i}}{\partial \epsilon_{p}}\right|_{\boldsymbol{\epsilon}=0}, \\
\xi_{\text {iap }}(\mathbf{X}, \mathbf{x}, \mathbf{P})=\left.\frac{\partial \bar{P}_{i a}}{\partial \epsilon_{p}}\right|_{\boldsymbol{\epsilon}=0} .
\end{gathered}
$$


We can obtain the following relation, which should be satisfied by the infinitesimal generators for the functional to remain infinitesimally invariant [17]. Consider a class of Lagrangians denoted by $L=L(\mathbf{x}, \mathbf{P}, \nabla \mathbf{P})$. We then have

$$
\frac{\partial L}{\partial X_{i}} \varphi_{i p}+\frac{\partial L}{\partial x_{i}} \lambda_{i p}+\frac{\partial L}{\partial P_{i a}} \xi_{i a p}+\frac{\partial L}{\partial P_{i a, b}}\left(\frac{d \xi_{i a p}}{d X_{b}}-P_{i a, c} \frac{d \varphi_{c p}}{d X_{b}}\right)+L \frac{d \varphi_{a p}}{d X_{a}}=0,
$$

where $i, a, b, c \in\{1,2,3\}$ and $p \in\{1,2, \ldots, r\}$. Further we can obtain the various conservation laws associated with invariant transformations as

$$
\frac{d}{d X_{a}}\left[\left(L \delta_{a b}-P_{i c, b} \frac{\partial L}{\partial P_{i c, a}}\right) \varphi_{b p}+\frac{\partial L}{\partial P_{i c, a}} \xi_{i c p}\right]=0 .
$$

In the case of Levinson's principle, $L \equiv-\left[\tilde{\Sigma}^{c}(\mathbf{P})+\mathbf{x} \cdot(\nabla \cdot \mathbf{P})\right]$. Under rigid body translations, $\bar{X}_{a}=X_{a}+\epsilon_{a}, \bar{x}_{i}=x_{i}$ and $\bar{P}_{i a}=P_{i a}$, the only non-trivial infinitesimal generator is $\varphi_{a b}=\delta_{a b}$ (therefore (62) is satisfied). Substituting it in (63) we obtain a dual energy momentum tensor from the Levinson's variational principle

$$
\mathcal{L}_{L}=-\tilde{\Sigma}^{c}(\mathbf{P}) \mathbf{1}+\mathbf{x} \cdot \nabla \mathbf{P}
$$

where we have made use of equilibrium condition $\nabla \cdot \mathbf{P}=0$, which arises as a one of the Euler equations of the Levinson's variation principle. In the rest of the paper, we simply denote $\mathcal{L}_{\mathrm{L}}$ as $\mathcal{L}$.

Another approach is to introduce a stress function $\Psi$ such that $P_{i a}=\varepsilon_{a b c}$ $\Psi_{i c, b}$. Due to such a substitution, the equilibrium condition is satisfied identically. We then have an alternative variational principle,

$$
\tilde{\Pi}^{c}(\nabla \Psi)=-\int_{\Omega} \tilde{\Sigma}^{c}(\mathbf{P}(\nabla \Psi)) d V+\int_{\Gamma_{u}} \mathbf{P}(\nabla \Psi) \mathbf{N} \cdot \hat{\mathbf{x}} d A .
$$

Therefore the Lagrangian under consideration is $L=L(\nabla \Psi)$. To obtain conservation laws for such a Lagrangian we consider the following r-parameter transformations:

$$
\begin{aligned}
\bar{X}_{a} & =\bar{X}_{a}(\mathbf{X}, \Psi, \boldsymbol{\epsilon}), \\
\bar{\Psi}_{i a} & =\bar{\Psi}_{i a}(\mathbf{X}, \Psi, \boldsymbol{\epsilon}),
\end{aligned}
$$

and the corresponding infinitesimal generators

$$
\begin{gathered}
\varphi_{a p}(\mathbf{X}, \Psi)=\left.\frac{\partial \bar{X}_{a}}{\partial \epsilon_{p}}\right|_{\boldsymbol{\epsilon}=0}, \\
\zeta_{i a p}(\mathbf{X}, \Psi)=\left.\frac{\partial \bar{\Psi}_{i a}}{\partial \epsilon_{p}}\right|_{\boldsymbol{\epsilon}=0} .
\end{gathered}
$$


The invariant conditions for the Lagrangian $L(\nabla \Psi)$ can be derived as [17]

$$
\frac{\partial L}{\partial X_{i}} \varphi_{i p}+\frac{\partial L}{\partial \Psi_{i a}} \zeta_{i a p}+\frac{\partial L}{\partial \Psi_{i a, b}}\left(\frac{d \zeta_{i a p}}{d X_{b}}-\Psi_{i a, c} \frac{d \varphi_{c p}}{d X_{b}}\right)+L \frac{d \varphi_{a p}}{d X_{a}}=0 .
$$

The conservation laws are given as

$$
\frac{d}{d X_{a}}\left[\left(L \delta_{a b}-\Psi_{i c, b} \frac{\partial L}{\partial \Psi_{i c, a}}\right) \varphi_{b p}+\frac{\partial L}{\partial \Psi_{i c, a}} \zeta_{i c p}\right]=0 .
$$

Let $\bar{X}_{a}=X_{a}+\epsilon_{a}$ and $\bar{\Psi}_{i a}=\Psi_{i a}$ (condition (70) is satisfied). We then obtain a dual energy momentum tensor,

$$
\tilde{\mathcal{L}}=-\tilde{\Sigma}^{c}(\mathbf{P}) \delta_{a p}+\Psi_{i c, p}\left(F_{i b} \varepsilon_{b a c}\right) .
$$

With some further algebraic manipulation, it can be shown that (72) differs from (64) by a divergent free quantity $\left[\left(\Psi_{i c, p} x_{i} \varepsilon_{a b c}\right)_{b}\right]$.

REMARK 3.2 The Eshelby's energy momentum tensor is given as [12]

$$
\mathcal{P}=\Sigma(\mathbf{F}) \mathbf{1}-\mathbf{F}^{T} \mathbf{P} .
$$

Subtracting (64) and (73), and on using the relation (34) we observe that

$$
\mathcal{P}-\mathcal{L}=(\mathbf{P}: \mathbf{F}) \mathbf{1}-\nabla(\mathbf{P} \cdot \mathbf{x}) .
$$

REMARK 3.3 Some further relations can be obtained from (64) and (73). We note that

$$
\begin{aligned}
& \operatorname{tr} \mathcal{L}=-3 \tilde{\Sigma}^{c}(\mathbf{P}), \\
& \operatorname{tr} \mathcal{P}=2 \Sigma(\mathbf{F})-\tilde{\Sigma}^{c}(\mathbf{P}),
\end{aligned}
$$

where $\operatorname{tr}$ represents the usual trace operator. The above observation can be helpful in understanding the physical nature of configurational forces and their relation to the energy densities. The above equations also imply that the energy densities can be in turn expressed as linear combinations of the Eshelby's energy momentum tensor and its dual counterpart.

REMARK 3.4. We can also express the functional (52) as a function of displacement $\mathbf{u}$ instead of the spatial coordinate $\mathbf{x}$. For such a case the Lagrangian is written as $L \equiv-\left[\tilde{\Sigma}^{c}(\mathbf{P})-\operatorname{tr} \mathbf{P}+\mathbf{U} \cdot(\nabla \cdot \mathbf{P})\right]$ and we obtain the following conservation law (corresponding to translational invariance):

$$
\hat{\mathcal{L}}=-\tilde{\Sigma}^{c}(\mathbf{P}) \mathbf{1}+(\operatorname{tr} \mathbf{P}) \mathbf{1}+\mathbf{U} \cdot \nabla \mathbf{P} .
$$

The above conservation law reduces to Bui's dual conservation law for the case of linear elastostatics. To see this, we first note that for the linear case $\tilde{\Sigma}^{c}(\sigma)=$ 
ON DUAL CONFIGURATIONAL FORCES

$\sigma: \nabla \mathbf{u}-\Sigma(\nabla \mathbf{u})$ and $\mathbf{P}$ can be replaced by $\sigma$. We can now rewrite (76) using $\mathbf{F}=\mathbf{1}+\nabla \mathbf{u}$ and (34) as

$$
\hat{\mathcal{L}}=-\tilde{\Sigma}^{c}(\sigma) \mathbf{1}+\mathbf{u} \cdot \nabla \sigma,
$$

which matches the corresponding expression in the infinitesimal case.

REMARK 3.5. The integral given by (65) is invariant if we add an arbitrary constant tensor to $\Psi_{i a}$. If we consider such a transformations with $\varphi_{a p}=0$, then the resulting conservation law gives us the integrability condition $\nabla \times \mathbf{F}=0$ in $\Omega$.

\subsection{THE FRAEIJS DE VEUBEKE PRINCIPLE}

To use Levinson's variational principle, invertibility of $\mathbf{P}(\mathbf{F})$ was assumed a priori. In case of lacking such a property, we can use complementary strain energy density defined by (37) and a stress function $\Psi$ such that $P_{i a}=\varepsilon_{a b c} \Psi_{i c, b}$ to write the following functional [32]:

$$
\check{\Pi}_{F}^{c}(\nabla \Psi, \mathbf{R})=-\int_{\Omega} \tilde{\Sigma}^{c}[\mathbf{T}(\nabla \boldsymbol{\Psi}, \mathbf{R})] d V+\int_{\Gamma_{u}} \mathbf{P}(\nabla \Psi) \mathbf{N} \cdot \hat{\mathbf{u}} d A .
$$

Therefore the Lagrangian under consideration is $L=L(\nabla \boldsymbol{\Psi}, \mathbf{R})$. To obtain conservation laws for such a Lagrangian we consider following r-parameter transformations:

$$
\begin{aligned}
& \bar{X}_{a}=\bar{X}_{a}(\mathbf{X}, \Psi, \mathbf{R}, \boldsymbol{\epsilon}), \\
& \bar{\Psi}_{i a}=\bar{\Psi}_{i a}(\mathbf{X}, \Psi, \mathbf{R}, \boldsymbol{\epsilon}), \\
& \bar{R}_{i a}=\bar{R}_{i a}(\mathbf{X}, \Psi, \mathbf{R}, \boldsymbol{\epsilon}),
\end{aligned}
$$

and their corresponding infinitesimal generators

$$
\begin{gathered}
\varphi_{a p}(\mathbf{X}, \boldsymbol{\Psi}, \mathbf{R})=\left.\frac{\partial \bar{X}_{a}}{\partial \epsilon_{p}}\right|_{\boldsymbol{\epsilon}=0}, \\
\zeta_{i a p}(\mathbf{X}, \boldsymbol{\Psi}, \mathbf{R})=\left.\frac{\partial \bar{\Psi}_{i a}}{\partial \epsilon_{p}}\right|_{\boldsymbol{\epsilon}=0}, \\
\eta_{i a p}(\mathbf{X}, \Psi, \mathbf{R})=\left.\frac{\partial \bar{R}_{i a}}{\partial \epsilon_{p}}\right|_{\boldsymbol{\epsilon}=0} .
\end{gathered}
$$


The invariant conditions for the Lagrangian $L(\nabla \Psi)$ are ([17])

$$
\left.\frac{\partial L}{\partial X_{i}} \varphi_{i p}+\frac{\partial L}{\partial \Psi_{i a}} \zeta_{i a p}+\frac{\partial L}{\partial R_{i a}} \eta_{i a p}+\frac{\partial L}{\partial \Psi_{i a, b}}\left(\frac{d \zeta_{i a p}}{d X_{b}}-\Psi_{i a, c} \frac{d \varphi_{c p}}{d X_{b}}\right)\right\}+L \frac{d \varphi_{a p}}{d X_{a}}=0 .
$$

The conserved quantities are then given as

$$
\frac{d}{d X_{a}}\left[\left(L \delta_{a b}-\Psi_{i c, b} \frac{\partial L}{\partial \Psi_{i c, a}}\right) \varphi_{b p}+\frac{\partial L}{\partial \Psi_{i c, a}} \zeta_{i c p}\right]=0 .
$$

Consider the admissible transformations $\bar{X}_{a}=X_{a}+\epsilon_{a}, \bar{\Psi}_{i a}=\Psi_{i a}$ and $\bar{R}_{i a}=R_{i a}$, i.e., the transformation satisfying (85). We obtain a dual energy-momentum tensor as

$$
\mathcal{L}_{\mathrm{F}}=-\tilde{\Sigma}^{c}(\mathbf{T}) \delta_{a p}+\Psi_{i c, p} F_{i b} \varepsilon_{b a c},
$$

which is similar to what we had obtained using Levinson's variational principle.

REMARK 3.6. All the remarks made at the end of previous section are valid here, except for the fact that the complementary energy density is now expressed as a function of the Biot tensor $\mathbf{T}$.

\subsection{THE GAO PRINCIPLE}

If the strain energy function $\Sigma(\mathbf{F})$ is non-convex, Levinson's principle becomes invalid. The Legendre-Fenchel transformation has to be then used in defining the complementary energy. Moreover, as pointed out by Gao [6, 7], there may exist duality gaps between the potential energy and the complementary potential energy for all three complementary variational principles (i.e., the HellingerReissner, the Levinson, and the Fraeijs de Veubeke). For Levinson's principle this duality gap is due to the fact that

$$
\inf _{\mathbf{u} \in \mathcal{U}_{\alpha}} \Pi(\mathbf{u}) \neq \sup _{\mathbf{P} \in \mathcal{T}_{\alpha}} \Pi_{L}^{c}(\mathbf{P}),
$$

where $\mathcal{U}_{a}=\left\{\mathbf{u} \in \mathcal{U} \mid \operatorname{det}(\mathbf{1}+\nabla \mathbf{u}(\mathbf{X}))>0 \forall \mathbf{X} \in \Omega, \mathbf{u}(\mathbf{X})=\mathbf{0} \forall \mathbf{X} \in \Gamma_{u}\right\}$ and $\mathcal{U}$ is the general admissible displacement field set. Also if $\mathcal{M}$ denotes the linear space of all real valued second-order tensors, then $\mathcal{T}_{\alpha}=\left\{\mathbf{P} \in \mathcal{M} \mid \nabla \cdot \mathbf{P}^{T}=\mathbf{0}\right.$ $\left.\forall \mathbf{X} \in \Omega, \mathbf{P} \cdot \mathbf{N}=\hat{\mathbf{t}}_{N} \forall \mathbf{X} \in \Gamma_{t}\right\}$.

Both the Hellinger-Reissner principle and the Fraeijs de Veubeke principle are not 'the pure complementary variational principles' because the HellingerReissner principle involves the displacement field and the Fraeijs de Veubeke principle involves the rotation tensor, $\mathbf{R}$. Therefore, they are not genuine complementary variational principles in the sense that they involve variables other than the stress tensor. 
Gao [6] proposed a pure complementary energy variational principle, which only involved the stress fields,

$$
\Pi_{G}^{c}(\mathbf{S})=-\int_{\Omega}\left\{\Sigma^{c}(\mathbf{S})+\frac{1}{2} \operatorname{tr}\left[\mathbf{P} \cdot \mathbf{S}^{-1} \cdot \mathbf{P}^{T}-2 \mathbf{P}+\mathbf{S}\right]\right\} d V,
$$

where the complementary energy density is defined by the following LegendreFenchel transformation:

$$
\Sigma^{c}(\mathbf{S})=\sup _{\mathbf{E} \in \mathcal{M}}\{\mathbf{S}: \mathbf{E}-\Sigma(\mathbf{E})\}
$$

where $\mathbf{S}$ belongs to a space of symmetric second order tensors with non-zero determinant, which is the range of the constitutive mapping $D \Sigma(\mathbf{E})$. We denote $\mathcal{S}_{a}=\{\mathbf{S} \in \mathcal{S} \mid \operatorname{det} \mathbf{S} \neq 0, \forall \mathbf{x} \in \Omega\}$.

Gao [6] showed that if $(\overline{\mathbf{U}}, \overline{\mathbf{S}})$ is a critical point of $\Gamma$,

$$
\Gamma(\mathbf{u}, \mathbf{S})=\int_{\Omega}\left(\mathbf{S}: \mathbf{E}(\mathbf{u})-\Sigma^{c}(\mathbf{S})\right) d V-\int_{\Omega} \hat{\mathbf{b}} \cdot \mathbf{u} d V-\int_{\Gamma_{t}} \hat{\mathbf{t}} \cdot \mathbf{u} d A,
$$

then $\Pi_{G}^{c}(\mathbf{S})$ satisfies the complementary condition

$$
\Pi(\overline{\mathbf{u}})=\Pi_{G}^{c}(\overline{\mathbf{S}}),
$$

where

$$
\Pi(\mathbf{u})=\int_{\Omega} \Sigma(\mathbf{F}(\mathbf{u})) d V-\int_{\Omega} \hat{\mathbf{b}} \cdot \mathbf{u} d V-\int_{\Gamma_{t}} \hat{\mathbf{t}} \cdot \mathbf{u} d A .
$$

In other words, there is no duality gap between the potential energy and the 465 complementary potential energy at the critical point.

The Euler equation of the Gao principle is: $\delta \Pi_{G}^{c}(\overline{\mathbf{S}}, \delta \mathbf{S})=0 \forall \delta \mathbf{S} \in \mathcal{S}_{a}$,

$$
\overline{\mathbf{S}}\left(2 D \Sigma^{c}(\overline{\mathbf{S}})+\mathbf{1}\right) \overline{\mathbf{S}}=\mathbf{P}^{T} \mathbf{P},
$$

where $D \Sigma^{c}(\mathbf{S})=\partial \Sigma^{c} / \partial \mathbf{S}$ stands for the Gâteaux derivative of $\Sigma^{c}$ with respect to $\mathbf{S}$ and $\overline{\mathbf{S}}$ is the solution the dual variational problem.

It should be noted that the only variational field in the functional in (89) is the second Piola-Kirchhoff stress tensor, $\mathbf{S}$.

Under coordinate translation, the divergence equation obtained as a result of the Noether's theorem takes the form

$$
\frac{d}{d X_{a}}\left[\left(L_{G} \delta_{a b}-S_{d c, b} \frac{\partial L_{G}}{\partial S_{d c, a}}\right) \delta_{b p}\right]=\frac{\partial L_{G}}{\partial X_{a}},
$$


where the Lagrangian $L_{G}$ is the integrand in the functional (89). The term on the right hand side of the above equality is due to the presence of $\mathbf{P}(\mathbf{X})$ in $L_{G}$, and therefore it can be expanded as (using the identities $\mathbf{P}=\mathbf{F S}$ and $\nabla \cdot \mathbf{P}=0$ )

$$
\begin{aligned}
\frac{\partial L_{G}}{\partial X_{a}} & =\frac{\partial L_{G}}{\partial P_{i c}} P_{i c, a} \\
& =\left(-S_{a b}^{-1} P_{j a} \delta_{i j} \delta_{b c}+\delta_{i a} \delta_{a c}\right) P_{i c, a} \\
& =\left(\delta_{i c}-F_{i c}\right) P_{i c, a} \\
& =-u_{i, c} P_{i c, a} .
\end{aligned}
$$

Since $\nabla \cdot \mathbf{P}=0$ we can rewrite $u_{i, c} P_{i c, a}=\left(u_{i} P_{i c, a}\right)_{, c}$. Note that $\partial L_{G} / \partial S_{d c, a}=$ 0 , hence (95) can be simplified as the following:

$$
\frac{d}{d X_{a}}\left(L_{G} \delta_{a p}\right)=-\left(u_{i} P_{i a, p}\right)_{, a},
$$

or

$$
\frac{d}{d X_{a}}\left(L_{G} \delta_{a p}+u_{i} P_{i a, p}\right)=0 .
$$

This indicates that the source term in the right-hand side of (95) can be expressed as a divergence, which leads to a Bessel-Hagen type symmetry or a BesselHagen type translational invariance. Therefore we obtain a conserved quantity as follows:

$$
\mathcal{L}_{G}=L_{G} \delta_{a b} \mathbf{e}_{a} \otimes \mathbf{e}_{b}+u_{i} P_{i a, b} \mathbf{e}_{a} \otimes \mathbf{e}_{b} .
$$

or

$$
\mathcal{L}_{G}=L_{G} \mathbf{1}+\mathbf{u} \cdot \nabla \mathbf{P},
$$

which is the same as (76) up to a divergent free term.

REMARK 3.7. It should be noted that we have not discussed all the symmetries that a variational principle can afford. Therefore there will be other conservation laws corresponding to these other symmetries. In this paper, our main objective was to study the dual energy-momentum tensor and therefore we restricted our attention to translational symmetry only.

REMARK 3.8. Another way to derive the expression for configuration forces is via thermodynamical considerations (see for e.g., Coleman and Noll [2], Gurtin $[9,10])$. In the following we follow Gurtin [10] to briefly outline the process to obtain a dual configurational force. The details will be provided in a separate 
paper. We start by writing an appropriate form of the second law of thermodynamics using the Gibbs free energy $\Phi$ (the dual of the Helmhotz free energy) as

$$
\frac{d}{d t} \int_{R} \Phi d v \leq \int_{\partial R}[-(\dot{P} \mathbf{m}) \cdot \mathbf{x}-(\dot{\mathcal{L} \mathbf{m}}) \cdot \mathbf{X}] d a,
$$

where $\left({ }^{*}\right)$ represents the total time derivative and $\mathbf{m}$ is the unit normal to the boundary $\partial R(t)$ of the referential control volume $R(t)$. We have neglected body forces in the above relation. The working $\mathcal{W}(R(t))$ can therefore be written as

$$
\mathcal{W}(R)=\int_{\partial R}[-(\dot{P m}) \cdot \mathbf{x}-(\dot{\mathcal{L}} \mathbf{m}) \cdot \mathbf{X}] d a
$$

We can expand the first term in the working as

$$
(\dot{\mathbf{P m}}) \cdot \mathbf{x}=(\dot{\mathbf{P}})_{\text {expl }} \mathbf{m} \cdot \mathbf{x}+(\mathbf{x} \cdot \nabla \mathbf{P}) \mathbf{m} \cdot \mathbf{v},
$$

where $\left({ }^{(}\right)_{\text {expl }}$ represents the explicit time derivative and $\mathbf{v}=\partial \mathbf{X} / \partial t$. We have assumed $\dot{\mathbf{m}}=0$. Considering (103) and

$$
(\dot{\mathcal{L}}) \cdot \mathbf{X}=(\mathcal{L} \mathbf{m} \cdot \mathbf{X})-\mathcal{L} \mathbf{m} \cdot \mathbf{v},
$$

we can rewrite (102)

$$
\mathcal{W}(R)=\int_{\partial R}\left[-(\dot{\mathbf{P}})_{\text {expl }} \mathbf{m} \cdot \mathbf{x}-(\mathcal{L} \mathbf{m} \cdot \mathbf{X})+\{-(\mathbf{x} \cdot \nabla \mathbf{P})+\mathcal{L}\} \mathbf{m} \cdot \mathbf{v}\right] d a .
$$

Consider a change in the velocity field $\mathbf{v}$ such that its normal component remains unaltered. The invariance of the working under such a change (see [10] for details) and the arbitrariness of the normal component of the velocity field can be then used to obtain the following relation for the dual configurational force:

$$
\mathcal{L}=\Phi \mathbf{1}+\mathbf{x} \cdot \nabla \mathbf{P} .
$$

The equivalence between the above relation and the one given in (64) can be argued by noticing that $\Phi=-\tilde{\Sigma}^{c}$.

\section{Closure}

In this paper, we present a systematic approach for deriving dual configuration forces in elasticity. Some previously unknown expressions of dual configuration forces have been discovered for the first time. These relations may prove valuable in the mathematical theory of elasticity, and they could be useful in 
theoretical developments of defect mechanics, for instance, obtaining bounds for the crack tip parameter, forces on dislocations, etc.

\section{Acknowledgments}

The authors would like to thank referees' comments and suggestions. This work is supported by a grant from National Science Foundation (Grant No. CMS0239130), which is greatly appreciated.

\section{References}

1. H.D. Bui, Dual path independent integrals in the boundary-value problems of cracks. Eng. Fract. Mech. 6 (1974) 287-296.

2. B.D. Coleman and W. Noll, The thermodynamics of elastic materials with heat conduction and viscosity. Arch. Ration. Mech. Anal. 13 (1963) 167-178.

3. J.D. Eshelby, The force on an elastic singularity. Philos. Trans. R. Soc. 244(877) (1951) 87111.

4. J.D. Eshelby, The continuum theory of lattice defects. In: F. Seitz and D. Turnbull (eds.), Solid State Physics, Vol. 3. Academic, New York (1956) pp. 79-144.

5. D.Y. Gao, Duality Principles in Nonconvex Systems. Kluwer (2000).

6. D.Y. Gao, General analytic solutions and complementary variational principles for large deformation nonsmooth mechanics. Meccanica 34 (1999) 169-198.

7. D.Y. Gao, Perfect duality theory and complete solutions to a class of global optimization. Optimization 52 (2003) 467-493.

8. Z.H. Guo, The unified theory of variational principles in nonlinear elasticity. Arch. Mech. 32 (1980) 577-596.

9. M.E. Gurtin, The nature of configurational forces. Arch. Ration. Mech. Anal. 131 (1995) 67100.

10. M.E. Gurtin, Configurational Forces as Basic Concepts of Continuum Mechanics. Springer, Berlin Heidelberg New York (1999).

11. R. Kienzler and G. Herrmann, Mechanics in Material Space. Springer, Berlin Heidelberg New York (2001).

12. J.K. Knowles and E. Sternberg, On a class of conservation laws in linear and finite elastostatics. Arch. Ration. Mech. Anal. 44 (1972) 187-211.

13. M. Levinson, Complementary energy theorem in finite elasticity. J. Appl. Mech. 32(4) (1965) 826-828.

14. S. Li, On dual conservation laws in linear elasticity: Stress function formalism. Nonlinear Dyn. 36 (2004) 77-96.

15. S. Li, A. Gupta and X. Markenskoff, Conservation laws of linear elasticity in stress formulations. Proc. R. Soc. Lond., A 461 (2005) 99-116.

16. X. Li, Dual conservation laws in elastostatics. Eng. Fract. Mech. 29(2) (1988) 233-241.

17. J.D. Logan, Invariant Variational Principles, Vol. 138, Mathematics in Science and Engineering. Academic, New York (1977).

18. G.A. Maugin, Material Inhomogeneties in Elasticity. Springer, Chapman and Hall, London (1993).

19. G.A. Maugin, Material forces: Concepts and applications. Appl. Mech. Rev. 48(5) (1995) 247-285.

20. B. Moran and C.F. Shih, Crack tip and associated domain integrals from momentum and engergy balance. Eng. Fract. Mech. 27 (1987) 615-642. 
ON DUAL CONFIGURATIONAL FORCES

21. B. Moran and C.F. Shih, A general treatment of crack tip contour integrals. Int. J. Fract. 35 (1987) 295-310.

22. E. Noether, Invariante variationsprobleme. Göttinger Nachrichten, (Mathematisch-physikalische Klasse) 2 (1918) 235-257. (Transl. Transport Theory and Statistical Physics 1 (1971) 186-207.)

23. R.W. Ogden, A note on variational theorems in non-linear elastostatics. Math. Proc. Camb. Philos. Soc. 77 (1975) 609-615.

24. R.W. Ogden, Non-linear Elastic Deformations. Dover (1997).

25. P.J. Olver, Conservation laws in elasticity. I. General results. Arch. Ration. Mech. Anal. 85 (1984) 119-129.

26. P.J. Olver, Conservation laws in elasticity. II. Linear homogeneous elastostatics. Arch. Ration. Mech. Anal. 85 (1984) 131-160.

27. P.J. Olver, Applications of Lie Group to Differential Equations. Springer, Berlin Heidelberg New York (1986).

28. E. Reissner, On a variational theorem for finite elastic deformations. J. Math. Phys. 32(2-3) (1953) 129-135.

29. J.R. Rice, A path-independent integral and the approximate analysis of strain concentrations by notches and cracks. J. Appl. Mech. 35 (1968) 379-386.

30. S.X. Sun, Dual conservation laws in elastostatics. Int. J. Eng. Sci. 23 (1985) 1179-1186.

31. C. Trimarco and G.A. Maugin, Bui's path-independent integral in finite elasticity. Meccanica 30 (1995) 139-145.

32. B.F. Veubeke, A new variational principle for finite elastic displacements. Int. J. Eng. Sci. 10 (1972) 745-763.

33. K. Washizu, Variational Methods in Elasticity and Plasticity, Vol. II. Oxford University Press (1975). 